\title{
Effects of deep and shallow water running on spinal shrinkage
}

\author{
C N Dowzer, T Reilly, N T Cable
}

\begin{abstract}
Objectives-Running in water has the potential to decrease the compressive forces on the spine as the body is supported. The aim of the study was to determine the magnitude of this loss in stature compared with running on land.

Methods-Fourteen runners completed three 30 minute runs on separate days in deep water, shallow water, and on a motor driven treadmill. During the three conditions, runners exercised at $80 \%$ of their exercise mode specific peak oxygen consumption. Subjects rested in the Fowler position for 20 minutes before and after exercise. Measurements of changes in stature were taken before resting, before running, after 15 minutes of running, after 30 minutes of running, and after the postexercise rest in the Fowler position. Changes in stature were recorded using a stadiometer accurate to $0.01 \mathrm{~mm}$.

Results-Loss of stature values were 4.59 (1.48), $5.51(2.18)$, and $2.92(1.7) \mathrm{mm}$ (means (SD)) for running on the treadmill, and in shallow and deep water respectively. Running in deep water caused significantly lower creep than in the other trials $(p<0.05)$, with no difference between the shallow water and treadmill conditions. Loss of stature was greater in the first half of the run for all conditions $(p<0.05)$. Ratings of perceived exertion did not differ between the three exercise conditions.

Conclusion-Results support the use of deep water running for decreasing the compressive load on the spine.

(BrF Sports Med 1998;32:44-48)
\end{abstract}

Keywords: perceived exertion; spinal loading; stature; endurance running; water running

Endurance running places repetitive stress on the lower limbs and lower back. Compressive loading is inevitable during running as the feet impact with the ground 600-1200 times per $\mathrm{km}^{1}$, with each foot strike inducing ground reaction forces equivalent to 2-4 times the body weight. ${ }^{2}$ This impact is attenuated by the training shoe or transmitted directly to the leg and spine, resulting in an increase in axial loading. When the compressive load exceeds the osmotic pressure of the discal tissues, fluid is expelled from the intervertebral discs. The resultant loss in disc height is reflected in a loss in stature which has been referred to as spinal shrinkage. ${ }^{3}$
The magnitude of shrinkage can be measured using precision stadiometry. ${ }^{4}$ This measuring technique has been validated by Boocock et al. ${ }^{6}$ Several authors have examined the effect of running on spinal loading. Garbutt et al reported significant differences in response to three running speeds, concluding that loss of stature increased as a function of exercise intensity. Greater loss in stature occurred during the first half of the 30 minute run than the second half, values being 3.26 (2.78) and 2.12 (1.61) $\mathrm{mm}$ respectively. This supports the observations of Reilly et $a l^{\beta}$ that the greatest loss in stature occurred in the early part of the run irrespective of the training mode (interval or continuous). This diminished response of the spine to compressive loading is due to a cumulative loss of fluid from the intervertebral discs. ${ }^{9}$ This rate of reduction in disc height is referred to as "creep", with greater compressive loads accelerating the creep response. ${ }^{10} 11$

The greater loss of stature in the early part of exercise may in part be explained by the exponential approach of Althoff et al, ${ }^{12}$ who devised a method of stature measurement that involved predicting the natural course of change in stature derived from observations during a pre-test period. The resultant change in stature during the test period was determined as the difference between predicted height and actual height during the test period. This technique eliminates the spinal loading history of individual subjects as well as accommodating the natural diurnal change in stature. However, this technique for determining spinal loading was deemed unsuitable for the present study as testing was carried out over a short duration and very small diurnal loss in stature would be balanced between the experimental conditions.

Running in water is a potentially useful therapeutic modality for runners with leg and back problems, since the body is supported. The added buoyancy has the potential to decrease the compressive forces on the spine which are evident during running on land. ${ }^{13}$ Deep water running is used by both injured and healthy athletes and appears to provide specificity of training for runners without incurring the potentially harmful effects of weight bearing exercise. Running in deep water with the aid of a flotation device allows the head to remain above the water and helps in maintenance of an upright position. An upright posture with the trunk perpendicular to the running surface is the ideal running position, allowing mobility of the pelvis and lumbar spine. This position is also the most efficient for ventilation. ${ }^{14}$ This is important as the chest is already under increased strain during deep 


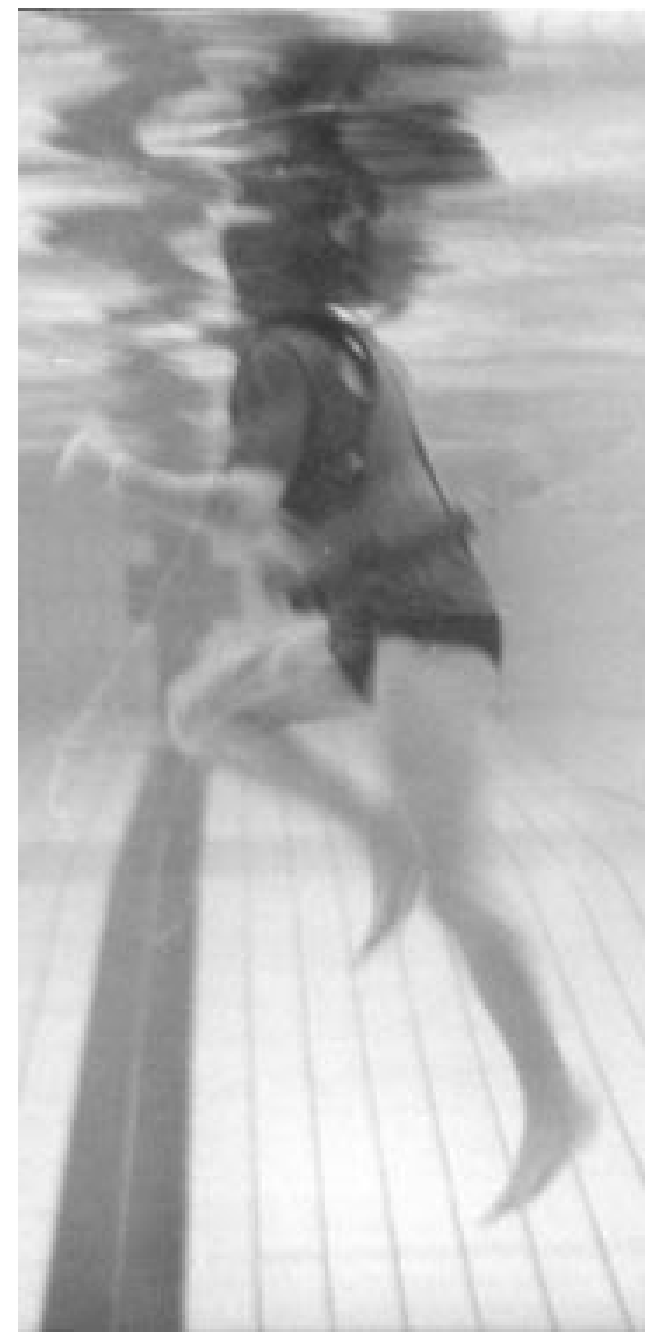

Figure 1 Subject in deep water running trial in the swimming pool.

water running because of the hydrostatic pressure of the water on the thoracic cavity. ${ }^{15}$ Shallow water running involves running in water at waist height with the arms remaining above water. Town and Bradley ${ }^{16}$ found that shallow water running produced greater physiological responses than deep water running in terms of the higher oxygen consumption $\left(\dot{\mathrm{V}}_{2}\right)(90.3$ and $73.5 \%$ of land values respectively) and heart rate $(88.6$ and $86 \%$ of land values respectively) attainable. Running in shallow water involves impact and incorporates a stance and push off phase of gait. Nevertheless shallow water running (and deep water running) alter the mechanics of running. In addition, the water resistance imposed on the body during aquatic locomotion is much greater than that on land, as water is about 800 times more dense than air. ${ }^{17}$ Furthermore, an additional resistance is generated by the water, with an increase in speed and surface area of the body. ${ }^{18}$

The effects of running in water on the compressive load of the spine have yet to be investigated. The aims of this study were to compare changes in stature induced by running on land, and in deep and shallow water.

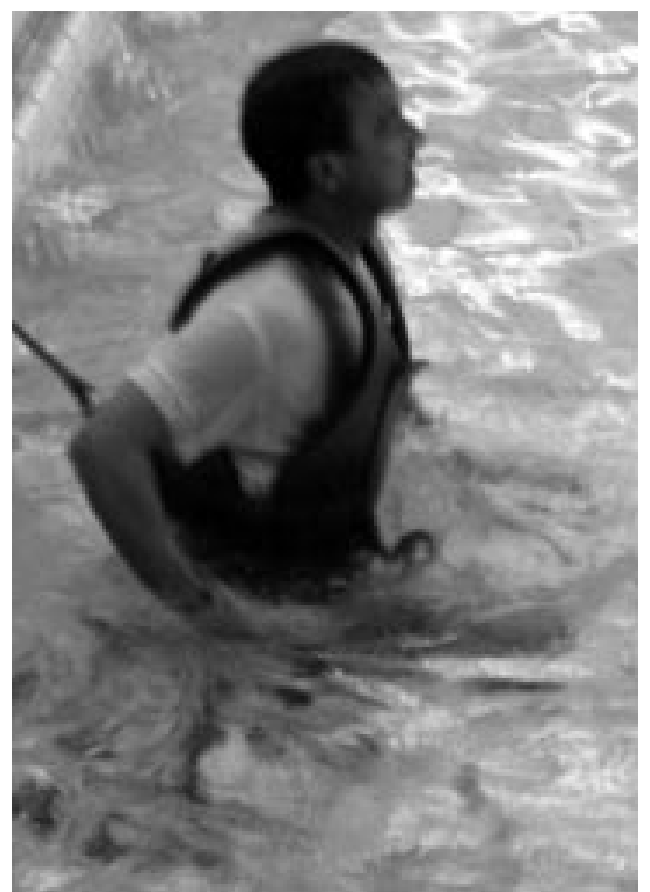

Figure 2 Subject in shallow water running trial in the swimming pool.

\section{Methods}

Fifteen male runners were recruited as a result of a questionnaire survey carried out on participants in endurance running events around the north west of England between March and May of 1995. Mean (SD) age, height, and mass for the group were 41.9 (9.1) years, $1.72(0.07) \mathrm{m}$, and $68.4(9.1) \mathrm{kg}$ respectively. Written informed consent to participate was obtained from all subjects, and the study was approved by the institution's human ethics committee.

Before measurement of the compressive loads on the spine, exercise intensity was determined in each of the three running media. This was established from maximal oxygen uptake tests carried out in each. Peak $\dot{V}_{2}$ values from the maximal exercise tests were 55.39 (8.46), $45.94(6.1)$, and $41.27(6.37) \mathrm{ml} / \mathrm{kg} / \mathrm{min}$ for treadmill, shallow water, and deep water running respectively (means (SD)). Exercise intensity for the spinal shrinkage study was set at $80 \%$ of mode specific peak $\dot{\mathrm{V}}_{2}$. Thus subjects were exercising at different absolute exercise intensities but the same relative intensity in each of the three running media.

The stadiometer used to measure changes in stature has previously been described by Boocock et al. ${ }^{6}$ A BBC microcomputer was used in combination with the apparatus to collect data. Before experimental testing, subjects were familiarised with the apparatus to improve reliability of subsequent experimental measurements. This also enabled the appropriate stadiometer settings to be established. A criterion of 10 consecutive measurements with a standard deviation of $0.5 \mathrm{~mm}$ or less was used to determine a subject's ability to obtain reproducible measurements of stature. On average, $1.8(0.6)$ training sessions were required in using the stadiometer. During training, 25.1 
Table 1 Changes in stature during a 30 minute run on a treadmill, in deep and shallow water (mean (SD))

\begin{tabular}{llll}
\hline & $\begin{array}{l}\text { Treadmill run } \\
(\mathrm{mm})\end{array}$ & $\begin{array}{l}\text { Shallow water } \\
\text { run (mm) }\end{array}$ & $\begin{array}{l}\text { Deep water run } \\
\text { (mm) }\end{array}$ \\
\hline After 20 min rest & $+2.52(1.85)$ & $+4.07(2.77)$ & $+3.21(1.65)$ \\
After first 15 min of running (80\% max) & $-3.35(1.48)^{\star}$ & $-4.52(2.15)^{\star}$ & $-1.85(1.48)$ \\
After second 15 min of running (80\% max) & $-1.24(0.99)$ & $-0.98(1.06)$ & $-1.07(0.93)$ \\
After 20 min recovery & $+2.83(1.23)$ & $+3.89(2.35)$ & $+2.25(1.53)$ \\
\hline${ }^{\star}$ p<0.05 difference in spinal shrinkage between first and second halves of the run. \\
Negative sign indicates a loss in stature, positive sign indicates an increase in stature.
\end{tabular}

(9.0) measures of stature lasting five seconds each were made, with the standard deviation for the last 10 consecutive measurements being 0.35 (range $0.19-0.49$ ) $\mathrm{mm}$. Training of subjects on the stadiometer was completed within 15-43 minutes. One subject who did not meet the criterion was omitted from the study, the remaining 14 subjects all meeting the criterion.

Subjects were asked to limit the intensity and duration of their exercise the day before testing by not performing speed work or running for longer than 30 minutes. Subjects were also instructed not to run on the morning of experimental testing, to prevent any possible fatigue effects on stature. The subjects undertook three experimental trials on three separate days. The order of the three test conditions was counterbalanced to eliminate sequence bias. Each subject was tested between 07:00 and 10:00 hours to control for circadian variation in stature. ${ }^{35} 19$

The experimental protocol was based on previous research on spinal shrinkage. ${ }^{7}$ It consisted of a pre-exercise control period where the subjects rested in the Fowler position for 20 minutes, a posture advocated for regaining stature. ${ }^{5}$ In this position the trunk is supine with the upper legs at an angle of $45^{\circ}$ and the lower legs supported horizontally. This was followed by two consecutive 15 minute runs on a motor driven treadmill, or in deep (fig 1) or shallow(fig 2) $(1.2 \mathrm{~m}$ ) water at $80 \%$ of the subject's predetermined peak $\dot{\mathrm{V}}_{2}$ in that specific exercise modality. After the second 15 minute run, the subject adopted the Fowler position for a recovery period of 20 minutes. Five measurements of stature were made: (a) before the control period; (b) at the end of the control period; (c) after the first 15 minute run; (d) after the second 15 minute run; and (e) after a 20 minute recovery period. Five consecutive measurements were taken on each of these five measuring occasions; between the five consecutive measures the subject moved away from the stadiometer after the third measurement to reduce the potential for fatigue from continued adoption of the test posture. A two minute period was allowed before each measurement occasion for heel pad thickness to compress. ${ }^{20}$ Before each experimental session, five control measurements were taken. This enabled the position of the adjustable supports to be checked and gave the subject the opportunity to become familiar again with the procedure and apparatus.

For the water runs, subjects were fitted with a Wet Vest (Bioenergetics Inc, Pelham, Alabama, USA) which maintained the body 2

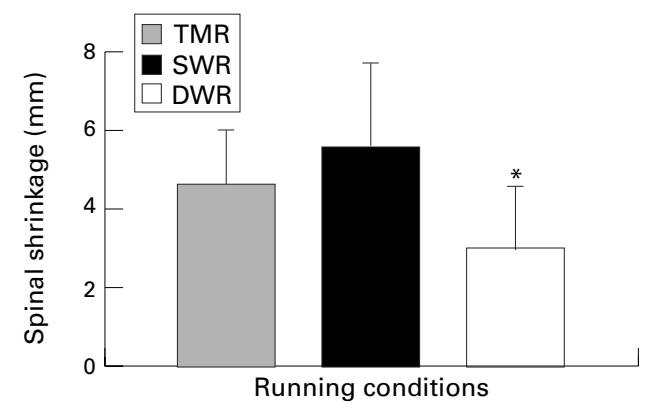

Figure 3 Mean losses ( $\pm S D$ ) in stature as a result of 30 minutes of running on a treadmill (TMR), and in shallow (SWR) and deep (DWR) water. ${ }^{*} p<0.05$ difference between deep water running and shallow water or treadmill running.

inches $(5.1 \mathrm{~cm})$ out of the water at the level of the chin and at waist height in the shallow water. During shallow water running, subjects wore aqua-pool shoes (Sprint Aqua pool shoe, Swim Shop, Luton, Beds, UK), providing traction and protecting the feet from any sharp tiles. Each subject was tethered about $1 \mathrm{~m}$ from the pool side with cord.

A metronome was used to pace each runner. The intensity of running was determined by identifying each subject's heart rate at $80 \%$ of peak $\mathrm{VO}_{2}$ in water and in motorised treadmill running. Heart rate was monitored during all three conditions using a short range radiotelemetry system (Sports Tester 3000: Kempele, Finland). A transmitter was worn at the base of the sternum at the xiphisternal joint and signals were transmitted to a receiver attached to the back of the Wet Vest in the water and to the treadmill on land. Borg's ${ }^{21}$ RPE scale was used for the subjects to rate the perceived exertion of their legs. Repeated measures analysis of variance and paired $t$ tests were employed in statistical analysis, with Tukey's post hoc test being used to locate differences among the means. A probability level of 0.05 was used to ascertain significance. A Pearson's product correlation analysis was used to assess the relationship between loss of stature and recovery of stature.

\section{Results}

Table 1 gives the effects of duration of the run on spinal shrinkage. The results show that duration of running has a significant effect on the shrinkage for the treadmill and shallow water conditions, with greatest shrinkage occurring in the first 15 minutes of each run $(p<0.05)$. No significant difference in shrinkage occurred between the first and second halves of the deep water run $(p>0.05)$. Figure 3 represents the shrinkage that occurred over the whole 30 minutes of running.

Running in deep water produced significantly less spinal shrinkage than either the treadmill or shallow water conditions $(p<0.05)$, no difference being evident between the treadmill and shallow water running. None of the correlations between total shrinkage for the whole run and subsequent recovery during deep water running $(r=0.59)$, shallow water running $(r=0.50)$ and treadmill running $(r=$ $0.07)$ reached significance. 
Mean (SD) ratings of perceived exertion for the first 15 minutes of the run were 3.6 (1.7), 3.6 (1.7), and 3.7 (1.9) for treadmill, shallow water and deep water running respectively. Ratings for the second 15 minutes of the run were 3.7 (2.0), 3.5 (1.8), and 4 (1.9) respectively. All numbers corresponded to "moderately hard" on the scale. No differences for perceived exertion were recorded between any of the three running conditions or between the subjective measures recorded after 15 and 30 minutes of running.

\section{Discussion}

The exercise intensity in each of the experimental trials corresponded to a fixed percentage of the prevailing peak $\dot{\mathrm{V}}_{2}$ established during maximal oxygen uptake tests in each training medium. As subjects were exercising at the same relative but not absolute exercise intensities for 30 minutes in the three running conditions, they were exercising at the same relative effort but at different absolute $\dot{\mathrm{V}}_{2}$ values. This was reflected in the rating of perceived exertion, which did not differ between the three exercise conditions. The results for spinal shrinkage indicate that greater compressive forces were incurred during treadmill and shallow water running than in deep water running. In this study the exercise intensity was matched to the subject's capability in the prevailing exercise, and so the relative metabolic strain (percentage of peak $\dot{\mathrm{V}}_{2}$ ) was consistent between exercise modes. Nevertheless, the absolute $\dot{\mathrm{VO}}_{2}$ in the deep water compared with the other two conditions was lower and could be associated with a reduced load on the spine. Thus it is not conclusive whether spinal shrinkage values were totally dependent on the exercise mode or were influenced in part by the absolute $\dot{\mathrm{V}}_{2}$ level. Further research is needed to determine whether metabolic responses per se affect the load on the spine. This could be achieved by assessing the stature changes associated with 30 minutes of treadmill running that elicit the same $\dot{\mathrm{V}}_{2}$ as during deep water running.

Although losses of stature for the shallow water condition were not significantly greater than for the treadmill run, shrinkage was greatest for 12 of the 14 subjects in the shallow water run condition. The rotation movement of the upper body which was subjectively observed during shallow water running to maintain balance may have contributed to the greater amount of shrinkage in this running mode and needs to be investigated further, possibly by measuring muscle forces acting on the spine.

No impact is made with the ground during deep water running. Thus it might be argued that the mechanism for inducing spinal shrinkage is absent. In the present study 30 minutes of running produced a loss of stature of 2.92 (1.7) $\mathrm{mm}$. Dolan \& Adams ${ }^{22}$ reported that the compressive loading due to body weight is about $380 \mathrm{~N}$ (55\% of body weight) when standing still. As total compressive forces acting on the lumbar discs during standing are about $500 \mathrm{~N},{ }^{22}$ the remainder must come from stabilisation of the upper body by the action of the back and abdominal muscles. The loss of stature that occurred during deep water running may be attributed to muscle tension in maintaining an upright posture in the water. Becker et $a l^{23}$ reported that flexion of the thoracolumbar spine was evident, regardless of the type of flotation device worn by the subjects, a posture that is discouraged during running on land. Excessive forward flexion of the lumbar spine was noted during the present study, the pelvis being tilted anteriorly. This places greater stress on the muscles of the back to maintain posture. In addition, the muscles of the back (such as the psoas major) involved in flexion of the hip have been shown to exert considerable compressive forces, which increase from L1-L2 down to L5-S1. ${ }^{24}$ Thus, during the running action when the psoas major is helping to coordinate movement of the spine with the legs, loss of stature may be incurred.

Greater losses of stature were observed for the first half of the run than the second, supporting previous research. ${ }^{78}$ The values may in part be due to the previous unloading of the spine in the Fowler position. A recovery of disc height when in the Fowler position is a result of a reduction in the compressive loading of the spine and the subsequent rehydration of the intervertebral discs. Thus the potential of the spine to undergo compression is increased after a period of unloading. This could explain why spinal shrinkage values were greater during the first half of the run than in the second half when creep effects within the discs are less responsive than before.

Several authors have examined the in vitro response of the intervertebral discs, showing that the discs respond in a viscoelastic manner. ${ }^{9}{ }^{10}{ }^{25}$ During periods of extended loading, the rate of reduction in disc height is nonlinear, with the greatest loss occurring in the early stages of loading. ${ }^{25}$ The implication is that the discs are stiffer in the later parts of prolonged exercise and as a result less responsive to impact loading.

The stadiometer used in the present study controlled for changes in spinal curvature that may be associated with changes in stature. Articular cartilage deformations and flattening of the arches of the feet are potential sources for changes in stature. However, the magnitudes probably do not account for the changes observed in the present study.

In conclusion, this study showed that loss of stature was greater during treadmill and shallow water running than during deep water running. The rate of shrinkage decreased as the run progressed, which supports other results in the literature. ${ }^{78}$ The present study is the first of its kind to examine spinal shrinkage during aqua-running and supports the use of deep water running for reducing the compressive load on the spine in both injured and uninjured runners. Analysis of spinal flexion during land and water running would provide a greater understanding of the loads being placed on the spine. This could be carried out with the use of a waterproof goniometer placed vertically along the spine. The findings of the present 
study clearly show that running in deep water markedly decreases spinal loading.

1 Valiant, GA. Transmission and attenuation of heel strike accelerations. In: Cavanagh P R, ed. Biomechanics of distance accelerations. In: Cavanagh P R, ed. Biomechanics of distance running. Cham

2 Cavanagh PR, Lafortune MA. Ground reaction forces in distance running. F Biomech 1980;13:397-406.

3 Reilly T, Tyrrell A, Troup JDG. Circadian variation in human stature. Chronobiol Int 1984;1:121-6.

4 Eklund JA, Corlett EN. Shrinkage as a measure of the effect of loading on the spine. Spine 1984;9:189-94

5 Tyrrell AR, Reilly T, Troup JDG. Circadian variation in stature and the effects of spinal loading. Spine 1985;10: $161-4$.

6 Boocock M, Reilly T, Linge K, Troup JDG. In: Reilly T, Watkins J, Borms J, eds. Fine measurement of stature for studying spinal loading. Kinanthropometry III. London: $\mathrm{E}$ and F N Spon, 1986;98-103.

7 Garbutt G, Boocock MG, Reilly T, Troup JDG. Running speed and spinal shrinkage in runners with and without low back pain. Med Sci Sports Exerc 1989;22:769-72.

8 Reilly T, Boocock MG, Garbutt G, Troup JDG. Shrinkage in total body length: its measurement and application. in total body length: its measurement and application. Humanbiology Budapest 1988,18:183-91.

9 Kazarian LE. Creep characteristics of the human spinal column. Orthop Clin North Am 1975;6:3-18.

10 Hirch C. The reaction of intervertebral discs to compression forces. F Bone foint Surg 1955;37:1118-96.

11 Troup JDG, Edwards FC. Manual handling: a review paper. London: HMSO, 1985.

12 Althoff I, Brinckmann, P, Frobin, W, et al. An improved method of stature measurement for quantitative determination of spinal loading: application to sitting postures and whole body vibration. Spine 1992;17:682-93.
13 Leatt P, Reilly T, Troup, JDG. Spinal loading during circuitweight training and running. Br F Sports Med 1986;20:11924 .

14 Slocum DB, James SL. Biomechanics of running. $7 A M A$ 1968;205:721-8.

15 Agostoni EG, Gurtner G, Torri G, Rahn H. Respiratory mechanics during submersion and negative-pressure breathing. f Appl Physiol 1966;21:251-8.

16 Town GP, Bradley SS. Maximal metabolic responses of deep and shallow water running in trained runners. Med Sci Sports Exerc 1991;23:238-41.

17 di Pampero, PE. The energy cost of human locomotion on land and in water. Int $\mathcal{F}$ Sports Med 1986;7:55-72.

18 Shanebrook J, Jaszczak R. Aerodynamic drag analysis of runners. Med Sci Sports 1976;8:43-5.

19 Wilby J, Linge K, Reilly T, Troup JDG. Spinal shrinkage in females: circadian variation and the effects of circuit weight-training. Ergonomics 1987;30:47-54.

20 Foreman TK, Linge K. The importance of heel compression in the measurement of diurnal stature variation. Applied Ergonomics 1989;20:299-300.

21 Borg GAV. Perceived exertion as an indicator of somatic stress. Scand f Rehabil Med 1970;2:92-8.

22 Dolan P, Adams MA. Forces acting on the lumbar spine. In: Aspden RM, Porter RW, eds. Lumbar spine disorders: current concepts. Singapore: World Scientific Publishing Co., 1995; 15-25.

23 Becker DG, Abidin MR, Lombardi SA, et al. Evaluation of flotation devices for deep-water exercise. F Burn Care Rehabil 1988;9:407-12.

24 Bogduk N, Pearcy M, Hadfield G. Anatomy and biomechanics of psoas major. Clin Biomech 1992;7:109-19.

25 Koeller W, Funke F, Hartmann F. Biomechanical behavior of human intervertebral discs subjected to long lasting axial loading. Biorheology 1984;21:675-86.

\section{Macleod's guidelines for doctors in sport: role and responsibilities of doctors in sport and exercise medicine}

- Know the participants and rules of the game as well as any associated patterns of injury, illness, or hazards.

- Clarify your relationship with players, officials, management, media, sponsors, and the public.

- Clarify your responsibilities for drug testing and the crowd.

- Establish a good records system. This may range from pre-participation medical examinations to documentation of injuries, illness, investigations, and rehabilitation programmes.

- Identify and minimise risk. This should include the climatic and playing environment; poor training and playing techniques or equipment; inappropriate attitudes and lack of respect for officials or opponents.

- Ensure that you are supported by appropriately experienced, qualified, and insured personnel and that facilities for rescue, first aid, treatment, transport to hospital, and communications are available.
- Prepare yourself in professional terms for responsibilities equivalent to those of an appointment to a post in an accident and emergency department combined with a physically and psychologically demanding industry - that is, sport and exercise.

- Join an appropriate specialty association, attend relevant training programmes, consider obtaining a higher degree or diploma, and maintain appropriate continuing professional development.

- Ensure that you are adequately insured, especially if you are not a general practitioner.

The doctor/patient relationship and player safety must remain your principal concern. Do no harm and minimise the risk of harm.

These guidelines are based on an article first published in the 1997 autumn edition of the Fournal of the Medical and Dental Defence Union of Scotland.

D A D MACLEOD West Lothian 